8 a Department of Medicine, Surgery and Veterinary Anatomy, University of León, León, Spain 9 Q1 ${ }^{\mathrm{b}}$ INDEGSAL and Molecular Biology, University of León, León, Spain

\section{Seminal plasma applied post-thawing affects boar sperm physiology: A flow cytometry study}

10

\section{Article history:}

Received 5 February 2013

Accepted 1 May 2013

\section{Keywords:}

Boar

Semen cryopreservation

Seminal plasma

Sperm physiology

Flow cytometry

\section{Introduction}

\section{A R T I C L E I N F O}

Received in revised form 28 April 2013

The achievement of successful semen cryopreservation would represent a dramatic leap in swine production systems. This would allow to the widespread use of germplasm banks for storing semen doses from selected males, for preserving genetic diversity, and for conserving rare breeds. Moreover, the long-term availability of frozen semen doses would allow for more flexible and efficient breeding programs, and it would help to control the

0093-691X/\$ - see front matter @ 2013 Elsevier Inc. All rights reserved. http://dx.doi.org/10.1016/j.theriogenology.2013.05.003

\begin{abstract}
A B S T R A C T
Cryopreservation induces extensive biophysical and biochemical changes in the sperm. In the present study, we used flow cytometry to assess the capacitation-like status of frozen-thawed boar spermatozoa and its relationship with intracellular calcium, assessment of membrane fluidity, modification of thiol groups in plasma membrane proteins, reactive oxygen species (ROS) levels, viability, acrosomal status, and mitochondrial activity. This experiment was performed to verify the effect of adding seminal plasma on post-thaw sperm functions. To determine these effects after cryopreservation, frozen-thawed semen from seven boars was examined after supplementation with different concentrations of pooled seminal plasma $(0 \%$, $10 \%$, and $50 \%$ ) at various times of incubation from 0 to 4 hours. Incubation caused a decrease in membrane integrity and an increase in acrosomal damage, with small changes in other parameters $(\mathrm{P}>0.05)$. Although $10 \%$ seminal plasma showed few differences with $0 \%$ (ROS fluidity increased considerably from the beginning of the experiment, and ROS and free thiols in the cell surface increased by 2 hours of incubation. By the end of the experiment, viability of $50 \%$ of seminal plasma seems to modify the physiology of thawed boar spermatozoa, possibly through membrane changes and ROS increase. Although some effects were detrithawed boar semen, as showed in the field (García JC, Domínguez JC, Peña FJ, Alegre B, Gonzalez R, Castro MJ, Habing GG, Kirkwood RN. Thawing boar semen in the presence of seminal plasma: effects on sperm quality and fertility. Anim Reprod Sci 2010;119:160-5). Q3
\end{abstract}

(c) 2013 Elsevier Inc. All rights reserved.

transmission of pathogens [1]. Despite the utilization of refrigerated long-term semen storage, the use of cryopreserved boar semen has not achieved widespread acceptability for commercial breeding by artificial insemination, mainly for economical and political reasons, although there is need for technical improvement too [2]. It is possible to achieve fertility results comparable to fresh semen by combining cryopreservation with deep intrauterine insemination and accurate estimation of ovulation [3]. However, the use of thawed semen results in decreased farrowing rates and litter size if the insemination occurs outside this ovulation time window. Environmental and management factors affect fertility of thawed semen more than when using refrigerated semen $[4,5]$. 
100 The damage to boar sperm caused by cryopreservation 101 includes motility impairment, chromatin damage, membrane 102 alterations, and decreased mitochondrial membrane poten103 tial, caused by cold shock, osmotic shock, and oxidative 104 damage by reactive oxygen species (ROS) to which boar 105 spermatozoa seem to be especially sensitive [6,7]. Several 106 factors have been presented as the cause of the low or 107 irregular fertility results of frozen-thawed boar spermatozoa, 108 including premature capacitation-like changes during the 109 process of cooling and cryopreservation [6,8]. These changes 110 have been termed as "cryocapacitation," and could shorten 111 the life span of spermatozoa, modify regulation pathways, 112 cause early acrosome reaction, and modify the plasma 113 membrane, resulting in part of the sperm population being unable to interact with the oviduct or to fertilize the ovum [9].

Using seminal plasma could help to improve semen quality after thawing. It is known that seminal plasma affects the physiology of spermatozoa, although its effects on different species are very variable, depending also on the condition of the sample [10-13]. In the case of boar spermatozoa, incubation of fresh or cryopreserved sperm in media supplemented with $10 \%$ seminal plasma seems to prevent, and possibly reverse, capacitation-related changes [7,8,14]. In several studies, the post-thawing addition of seminal plasma improved membrane and acrosomal integrity, and enhanced the in vivo fertilization [15]. Use of $10 \%$ seminal plasma after thawing rendered good fertility results when combined with a modified freezing/thawing protocol [16].

However Abad et al. [17] found that $10 \%$ seminal plasma supplementation did not affect the creation of the oviductal sperm reservoir. The same authors reported no improvement on sow fertility when thawed boar semen was supplemented with $10 \%$ seminal plasma and inseminated by 2 or 12 hours of the predicted time of ovulation [18]. Therefore, they concluded that seminal plasma could not completely reverse cryocapacitation, or else other factors were having a role, decreasing cryopreserved semen fertility.

The objective of the present study was to enhance our understanding on the effects of post-thawing addition of seminal plasma to boar semen by analyzing several physiological variables using flow cytometry. This study follows a previous trial in which adding 50\% seminal plasma to thawed boar semen made both pregnancy rate and mean litter size comparable to those achieved with liquid-stored semen [19]. Taking this study as a starting point, we posed the hypothesis that the seminal plasma would modify the physiology of thawed spermatozoa, explaining the fertility nprovement found by [19]. Thus, we assessed the sperlatozoa during a 4-hour incubation in the presence of $50 \%$ r aminal plasma. We also tested $10 \%$ seminal plasma, to include a concentration widely used in other studies but which has yielded mixed results.

\section{Materials and methods}

\subsection{Experimental design}

The spermatozoa used in this experiment were obtained from frozen semen doses stored in our cryobank. After thawing, the pooled contents of two straws were diluted down to $25 \times 10^{6} / \mathrm{mL}$ with MR-A extender (Kubus S.A.,
Madrid, Spain), split among three 1.5-mL tubes, and supplemented with $0 \%, 10 \%$, or $50 \%$ of heterologous seminal plasma. The tubes were incubated at $37^{\circ} \mathrm{C}$ and assessed each hour (sampling points at 0,1, 2, 3, and 4 hours). At each sampling point, an aliquot from each tube was mixed with fluorescence probes for assessing several physiological parameters: stability of the plasma membrane (damage, apoptotic-like changes, and fluidity), acrosomal damage, mitochondrial activity, intracellular $\mathrm{Ca}^{2+}$ concentration, intracellular ROS concentration, and abundance of extracellular free thiols.

\subsection{Reagents and media}

Fluo-4 AM cell permeant, merocyanine 540 (M540), propidium iodide (PI), 2' $7^{\prime}$-dichlorodihydrofluorescein diacetate (CM- $\mathrm{H}_{2}$ DCFDA), 5-iodoacetamidofluorescein (5-IAF) Mitotracker Deep Red, and YO-PRO-1 iodide used in the study were purchased from Invitrogen (Carlsbad, CA, USA). Other chemicals such as fluorescein isothiocyanate-peanut agglutinin (FITC-PNA), DMSO, and PBS were purchased from Sigma (St. Louis, MO, USA). Stock solutions of fluorescence probes were prepared in DMSO at $1 \mathrm{mM}$, except YOPRO-1 (25 $\mu \mathrm{M})$, PI ( $1 \mathrm{mg} / \mathrm{mL}$ in water), and PNA-FITC $(0.2$ $\mathrm{mg} / \mathrm{mL}$ in water). These stocks were kept at $-20{ }^{\circ} \mathrm{C}$ in the dark. Flow cytometry equipment, software, and consumables were purchased from Becton Dickinson (San Jose, CA, USA).

\subsection{Semen collection and preservation}

Semen was collected from seven mature Landrace, Large White, and Duroc boars by the "gloved-hand method." For each ejaculate, the sperm concentration was determined using a spectrophotometer. The initial percentage of motile sperm was determined visually and any ejaculates containing $>60 \%$ motile sperm were used.

Semen was processed for cryopreservation according to the technique described by Eriksson and Rodríguez-Martínez [20], except that our first extender dilution was MR-A at 2:1 rather than BTS at 1:1, and we used slower cooling and $\mathbf{Q 5}$ freezing curves [21]. Initially, $60 \times 10^{9}$ sperm from each ejaculate were diluted in MR-A previously warmed to $32.5{ }^{\circ} \mathrm{C}$. The extended semen was incubated at room temperature $\left(20^{\circ} \mathrm{C}-22^{\circ} \mathrm{C}\right)$ for 1 hour. Then, the semen was transferred to a room at $15{ }^{\circ} \mathrm{C}$ for 3 hours, centrifuged at $800 \times g$ for 10 minutes at $15^{\circ} \mathrm{C}$ in a programmable refrigerated centrifuge (Heraeus Megafuge 1.0 R, Heraeus Holding $\mathrm{GmbH}$, Germany), and the supernatant was discarded. The pellets were reextended with lactose-egg yolk extender ( $80 \%$ ( vol/vol) of a $11.0 \%$ (wt/vol) lactose monohydrate solution, and $20.0 \%$ ( $\mathrm{vol} / \mathrm{vol}$ ) of hen's egg yolk) to a final concentration of $1.5 \times 10^{9}$ spermatozoa/mL. After thorough mixing, the semen was cooled for 2 hours in a refrigerator at $5{ }^{\circ} \mathrm{C}$. At this temperature, the semen was slowly mixed with the freezing extender, consisting of $89.55 \%$ (vol/vol) lactoseegg yolk extender, $8.95 \%$ ( $\mathrm{vol} / \mathrm{vol}$ ) glycerol, and 1.5\% ( $\mathrm{vol} / \mathrm{vol}$ ) Equex STM (Minitüb, Germany) at a ratio of two parts of semen to one part of extender, yielding a final concentration of $3 \%$ glycerol and $1 \times 10^{9}$ spermatozoa $/ \mathrm{mL}$.

Sperm were packaged at $5{ }^{\circ} \mathrm{C}$ in $0.25 \mathrm{~mL}$ straws. After sealing, all racks were transferred to the chamber of 
222 a programmable freezer (Ice Cube 1810, Sy-Lab, Purkers-

dorf, Australia) set at $5{ }^{\circ} \mathrm{C}$. The cooling/freezing rate used was $3{ }^{\circ} \mathrm{C} /$ minutes from +5 to $-6^{\circ} \mathrm{C}$, hold for 1 minute for crystallization, and thereafter $-20^{\circ} \mathrm{C}$ /minute from $-6{ }^{\circ} \mathrm{C}$ to $-100^{\circ} \mathrm{C}$. Samples were then plunged into liquid nitrogen $\left(-196{ }^{\circ} \mathrm{C}\right)$ for storage. The straws were thawed in circulating water at $50{ }^{\circ} \mathrm{C}$ for 12 seconds. After thawing, samples were checked for motility (at least $>50 \%$ motile spermatozoa).

\subsection{Seminal plasma processing}

The seminal plasma was obtained after a double centrifugation $\left(800 \times g\right.$ for 10 minutes at $\left.25^{\circ} \mathrm{C}\right)$ of a semen pool derived from 11 boars (Duroc, Large White, and Landrace) and was stored at $-20{ }^{\circ} \mathrm{C}$ until needed. These boars were healthy, yielded semen of good quality, and were routinely used for preparing insemination doses, reportedly of high fertility. Thawing of seminal plasma was done at $37^{\circ} \mathrm{C}$ in a water bath.

\subsection{Assessment of sperm physiology by flow cytometry}

\subsubsection{Flow cytometer configuration}

Flow cytometric analyses were carried out on a FACScalibur (Becton Dickinson Immunochemistry Systems, San Jose, CA, USA). Green fluorescence from Fluo-4 AM, YOPRO-1, 5-IAF, and CM- $\mathrm{H}_{2}$ DCFDA was read with the FL1 photodetector (530/30BP filter). Orange fluorescence from merocyanine 540 was read with the FL2 photodetector (575/25BP filter). Red fluorescence from PI was read with the FL3 photodetector (670LP filter). Mitotracker Deep Red was excited using a 633-nm He-Ne laser, and its red fluorescence was read with the FL4 photodetector (670/40BP filter). Fluorescence data were displayed in logarithmic mode using the Cell Quest Pro 3.1 software (BD Biosciences). Ten thousand events were collected per sample, with a flow rate of 200 cells/second, using a gate in forward and side scatter to exclude debris and aggregates from the analysis. The analysis of the flow cytometry data was carried out using Weasel v. 3.0.1 (WEHI, Melbourne, Australia).

\subsubsection{Intracellular ROS}

$\mathrm{CM}-\mathrm{H}_{2}$ DCFDA was used for the detection of intracellular ROS. CM- $\mathrm{H}_{2}$ DCFDA is oxidized to dichlorofluorescein (DCF), which emits fluorescence at $530 \mathrm{~nm}$ in response to the 488$\mathrm{nm}$ excitation. This fluorescent probe was combined with PI for removing the nonviable population from the analysis [22]. Samples were prepared at $10^{6} / \mathrm{mL}$ in PBS with $1 \mu \mathrm{M}$ $\mathrm{CM}-\mathrm{H}_{2}$ DCFDA and $2.5 \mu \mathrm{g} / \mathrm{mL}$ PI. After 15 minutes at $37^{\circ} \mathrm{C}$ in the dark, the samples were analyzed. The median of the green fluorescence intensity was used for analysis, after excluding PI+ events (dead spermatozoa).

\subsubsection{Free thiols in the cell surface}

The free thiol groups of proteins from the sperm surface were evaluated by staining with 5-IAF. Samples were prepared at $10^{6} / \mathrm{mL}$ in PBS with $25 \mathrm{nM} 5$-IAF and $2.5 \mu \mathrm{g} / \mathrm{mL}$ PI. After 15 minutes at $37^{\circ} \mathrm{C}$ in the dark, the samples were analyzed. The median of the green fluorescence intensity was used for analysis, after excluding PI+ events (dead spermatozoa).

\subsubsection{Membrane fluidity of spermatozoa}

Merocyanine 540 (M540) binds preferentially to membranes with loosely packed lipids, whereas YO-PRO-1 stains the nuclei of cells with increased plasma membrane permeability ("apoptotic" or dead). Samples were prepared at $10^{6} / \mathrm{mL}$ in PBS with $2.7 \mu \mathrm{M}$ M540 and $0.1 \mu \mathrm{M}$ YO-PRO-1. After 15 minutes at $37{ }^{\circ} \mathrm{C}$ in the dark, the samples were analyzed. Spermatozoa belonged to one of three populations: high YO-PRO-1 fluorescence (dead or increased membrane permeability), low M540 fluorescence and low YO-PRO-1 fluorescence (lower membrane fluidity), and high M540 fluorescence and low YO-PRO-1 fluorescence (higher membrane fluidity).

\subsubsection{Intracellular $\mathrm{Ca}^{2+}$}

The state of the spermatozoa in terms of intracellular calcium content and membrane integrity was evaluated by combining PI and Fluo-4 AM, a probe that accumulates intracellularly and increases its green fluorescence on binding Ca. Samples were prepared at $10 \mathrm{~mL}$ in PBS with $10 \mathrm{nM}$ Fluo-4 AM and $2.5 \mu \mathrm{g} / \mathrm{mL}$ YO-PRO-1. After 15 minutes at $37{ }^{\circ} \mathrm{C}$ in the dark, the samples were analyzed. The median of the green fluorescence intensity was used for analysis, after excluding PI+ events (dead spermatozoa).

\subsubsection{Acrosomal integrity}

The integrity of the acrosome was estimated using the lectin PNA combined with FITC. Samples were prepared at $10^{6} / \mathrm{mL}$ in PBS with $10 \mu \mathrm{g} / \mathrm{mL}$ PNA-FITC and $2.5 \mu \mathrm{g} / \mathrm{mL}$ PI. After 15 minutes at $37{ }^{\circ} \mathrm{C}$ in the dark, the samples were analyzed. Spermatozoa were classified into viable, acrosome-intact (PNA-/PI-); viable, acrosome-damaged (PNA+/PI-); dead, acrosome-intact (PNA-/PI+); and dead, acrosome-damaged (PNA+/PI+). Data were expressed as the percentage of sperm in each category.

\subsubsection{Mitochondrial activity}

Sperm mitochondrial function was assessed using Mitotracker Deep Red. This dye is readily sequestered in mitochondria and emits red fluorescence in active mitochondria (high mitochondrial membrane potential). Samples were prepared at $10^{6} / \mathrm{mL}$ in PBS with $100 \mathrm{nM}$ Mitotracker Deep Red and $0.1 \mu \mathrm{M}$ YO-PRO-1. After 15 minutes at $37^{\circ} \mathrm{C}$ in the dark, the samples were analyzed.

\subsubsection{Plasma membrane permeability}

Variations of plasma membrane permeability were assessed using the YO-PRO-1/PI combination (PI identifies YO-PRO-1+ spermatozoa with extensive membrane damage). Samples were prepared at $10^{6} / \mathrm{mL}$ in PBS with $100 \mathrm{nM}$ YO-PRO-1 and $2.5 \mu \mathrm{g} / \mathrm{mL}$ PI. After 15 minutes at $37^{\circ} \mathrm{C}$ in the dark, the samples were analyzed. Spermatozoa were classified into viable, normal membrane permeability (YO-PRO-1-/PI-); viable, increased membrane permeability (YO-PRO-1+/PI-); and dead (YO-PRO-1+/PI+). Data were expressed as the percentage of spermatozoa in each category. 


\subsection{Statistical analysis}

The statistical analyses were carried out in the $R$ statistical environment [23]. Data were analyzed using linear mixed-effects models. Incubation time and seminal plasma concentration were analyzed as fixed factors, with the male as the grouping factor in the random part of the models. Results are presented as mean \pm SEM.

Moreover, to improve our understanding of the changes underwent within each treatment during the incubation, we carried out a multidimensional analysis of the data. Five variables (viability, membrane fluidity, acrosomal damage, free thiols, and intracellular ROS) were used in this study. Data were standardized and centered before performing a principal components analysis (PCA). The data points were plotted for each sampling time according to the two first principal components (selected using Kaiser's criterion). Thus, the observations could be studied considering the contribution of each original variable to each principal component (each linear component is the linear combination of each original variable), considering the plot as a multidimensional space. For facilitating the interpretation of these plots, vectors representing the contribution of each original variable to the principal components (axes) were overplotted, and we performed a clustering analysis to highlight the relationship between observations. We used an algorithm for agglomerative nesting processing (AGNES) [24], a kind of hierarchical clustering algorithm (using euclidean metric and Ward's clustering method). The selection of the final number of clusters $(k)$ was based on the "silhouette information," choosing such a $k$ that maximized the local "silhouette average width."

\section{Results}

The results showed that boar spermatozoa incubated with seminal plasma after thawing undergo profound changes in their physiology, compared with samples incubated in the same conditions without seminal plasma. These changes were evident when a high proportion of seminal plasma (50\%) was used.
Table 1 displays the variables related to the plasma decreased with incubation time in the three treatments, as expected, although this decrease was not dramatic in any case (overall, from $68.6 \% \pm 1.0 \%$ at 0 hours to $52.5 \% \pm 2.4 \%$ at 4 hours). Viability was similar, on average, among the three treatments, with a transient increase in samples incubated with $50 \%$ seminal plasma for 2 hours $(\mathrm{P}=0.006$ vs. $0 \%$ and $P=0.030$ vs. $10 \%$ ). At 4 hours, this group presented a significantly lower proportion of viable spermatozoa (a difference of $-13.1 \pm 3.6$ points respect to no seminal plasma). The proportion of spermatozoa showing altered membrane permeability without permeating PI (an apoptotic feature) was very low in all cases, with no evident trends or differences among treatments. Contrarily, the proportion of viable spermatozoa with increased membrane fluidity (higher merocyanine 540 staining) was considerably higher in the samples incubated with $50 \%$ seminal plasma. This increase was very fast, being noticeable just after adding the seminal plasma ( $P=0.090$ for $50 \%$ seminal plasma with respect to $0 \%$ ), leaping to $>30 \%$ after 1 hour and $>40 \%$ after 2 hours, whereas $0 \%$ and $10 \%$ samples remained stable $(\mathrm{P}<0.001)$. This increase was still more dramatic when presenting the data relative to the viable spermatozoa population. Although samples with $0 \%$ or $10 \%$ seminal plasma showed a slow and nonsignificant increase in the proportion of viable spermatozoa with increased membrane fluidity (always below 10\% on average), this proportion considerably rose with time in the samples incubated with $50 \%$ seminal plasma, showing that, on average, $>70 \%$ of viable spermatozoa showed increased membrane permeability at 4 hours.

These changes in membrane fluidity in the samples incubated with $50 \%$ seminal plasma reflected, at least in part, in the results on acrosomal damage (Table 2), intracellular ROS, and presence of free thiols on the sperm surface (Table 3). Most of the damaged acrosomes corresponded to spermatozoa that were considered "dead" (damaged membrane according to PI stain), which was evidenced by the low proportion of spermatozoa unstained by PI that were stained by PNA-FITC $(2.7 \% \pm 0.3 \%)$. The proportion of damaged acrosomes increased with time membrane. The viability (\% of YO-PRO-1- spermatozoa)

Plasma membrane status during the incubation with different proportions of seminal plasma.

\begin{tabular}{|c|c|c|c|c|c|c|}
\hline \multirow[t]{2}{*}{ Variable } & \multirow[t]{2}{*}{ Plasma } & \multicolumn{5}{|c|}{ Hours of incubation } \\
\hline & & 0 & 1 & 2 & 3 & 4 \\
\hline \multirow[t]{3}{*}{ Viability (\%) } & 0 & $69.2 \pm 1.7 \mathrm{a}^{\mathrm{a} \alpha}$ & $67.8 \pm 2.7^{\alpha . \beta}$ & $64.2 \pm 2.4^{\mathrm{a} \alpha \beta}$ & $61.8 \pm 3.6^{\beta \gamma}$ & $57.2 \pm 3.8^{\mathrm{a} \gamma}$ \\
\hline & 10 & $67.4 \pm 2^{\mathrm{b} \alpha}$ & $67.5 \pm 3.1^{\alpha}$ & $64.9 \pm 3.5^{\text {a } \alpha \beta}$ & $62.1 \pm 3.8^{\beta}$ & $56.1 \pm 2.1^{\mathrm{a \gamma}}$ \\
\hline & 50 & $69.2 \pm 1.8^{\mathrm{a} \alpha}$ & $68.9 \pm 1.9^{\alpha}$ & $68.2 \pm 2.7^{\mathrm{b} \alpha}$ & $60.4 \pm 3.5^{\beta}$ & $42.6 \pm 3.9^{\mathrm{b} \gamma}$ \\
\hline \multirow[t]{3}{*}{ Increased membrane permeability (\%) } & 0 & $1.6 \pm 0.7$ & $1 \pm 0.3$ & $0.8 \pm 0.3$ & $1.1 \pm 0.5$ & $1.5 \pm 0.7$ \\
\hline & 10 & $1.4 \pm 0.5$ & $1.1 \pm 0.4$ & $0.9 \pm 0.3$ & $1 \pm 0.4$ & $1.3 \pm 0.5$ \\
\hline & 50 & $1.7 \pm 0.6$ & $1.5 \pm 0.9$ & $0.8 \pm 0.4$ & $0.8 \pm 0.2$ & $1.5 \pm 0.7$ \\
\hline \multirow[t]{3}{*}{ Membrane fluidity (\%) } & 0 & $4.6 \pm 1.7$ & $3.5 \pm 0.4^{\mathrm{a}}$ & $4.2 \pm 0.9^{\mathrm{a}}$ & $4.6 \pm 1.4^{\mathrm{a}}$ & $5.3 \pm 1.7^{\mathrm{a}}$ \\
\hline & 10 & $4.5 \pm 1.6$ & $5.1 \pm 0.5^{\mathrm{a}}$ & $5.4 \pm 1.7^{\mathrm{a}}$ & $5.2 \pm 2.1^{\mathrm{a}}$ & $5.5 \pm 2.2^{\mathrm{a}}$ \\
\hline & 50 & $7.9 \pm 1.9^{\alpha}$ & $31.8 \pm 4.1^{\mathrm{b} \beta}$ & $42.7 \pm 2.3^{\mathrm{b} \beta}$ & $40 \pm 3.4^{\mathrm{b} \beta}$ & $35.8 \pm 3.4^{\mathrm{b} \beta}$ \\
\hline \multirow[t]{3}{*}{ Membrane fluidity (viable ratio) (\%) } & 0 & $6.8 \pm 2.6$ & $5.2 \pm 0.7^{\mathrm{a}}$ & $6.4 \pm 1.1^{\mathrm{a}}$ & $7.6 \pm 2.3^{\mathrm{a}}$ & $8.7 \pm 2.8^{\mathrm{a}}$ \\
\hline & 10 & $6.8 \pm 2.7$ & $7.6 \pm 0.8^{\mathrm{a}}$ & $8.3 \pm 2.5^{\mathrm{a}}$ & $8.6 \pm 3.6^{\mathrm{a}}$ & $9.5 \pm 4^{\mathrm{a}}$ \\
\hline & 50 & $11.6 \pm 2.9^{\alpha}$ & $46.4 \pm 6.8^{\mathrm{b} \beta}$ & $62 \pm 3.3^{\mathrm{b} \gamma}$ & $64.7 \pm 5^{\mathrm{b} \gamma}$ & $71.6 \pm 4.2^{\mathrm{b} \gamma}$ \\
\hline
\end{tabular}

Values are presented as mean \pm SEM, with significant differences among plasma concentrations (rows) indicated by Latin letters and significant differences among times (columns) indicated by Greek letters. 
Table 2

Organelle status and intracellular calcium concentration during incubation with different proportions of seminal plasma.

\begin{tabular}{|c|c|c|c|c|c|c|}
\hline \multirow[t]{2}{*}{ Variable } & \multirow[t]{2}{*}{ Plasma } & \multicolumn{5}{|c|}{ Hours of incubation } \\
\hline & & 0 & 1 & 2 & 3 & 4 \\
\hline \multirow[t]{3}{*}{ Damaged acrosomes (total) (\%) } & 0 & $22.4 \pm 2.5^{\alpha}$ & $25.8 \pm 2.1^{\mathrm{a} \alpha \beta}$ & $28.6 \pm 1.8^{\beta \gamma}$ & $31.1 \pm 2.5^{\mathrm{a} \gamma}$ & $32 \pm 2.5^{\mathrm{a} \gamma}$ \\
\hline & 10 & $23.4 \pm 2.3^{\alpha}$ & $27.2 \pm 2.3^{\mathrm{ab} \alpha \beta}$ & $29.6 \pm 1.7^{\beta \gamma}$ & $31.9 \pm 1.6^{\mathrm{a} \gamma \delta}$ & $34.4 \pm 1.5^{\mathrm{a} \delta}$ \\
\hline & 50 & $24.1 \pm 2.8^{\alpha}$ & $29.5 \pm 2.4^{\mathrm{b} \alpha \beta}$ & $31.7 \pm 3.3^{\beta \gamma}$ & $37.3 \pm 2.3^{\mathrm{b} \gamma \delta}$ & $43.1 \pm 3.2^{\mathrm{b} \delta}$ \\
\hline \multirow[t]{3}{*}{ Damaged acrosomes (viable) (\%) } & 0 & $1.2 \pm 0.3^{\mathrm{a}}$ & $1.1 \pm 0.3^{\mathrm{a}}$ & $0.9 \pm 0.2^{\mathrm{a}}$ & $0.9 \pm 0.1^{\mathrm{a}}$ & $0.9 \pm 0.2^{\mathrm{a}}$ \\
\hline & 10 & $1.6 \pm 0.5^{\mathrm{a}}$ & $1.8 \pm 0.4^{\mathrm{a}}$ & $2.2 \pm 0.7^{\mathrm{a}}$ & $2 \pm 0.5^{a}$ & $1.6 \pm 0.4^{\mathrm{a}}$ \\
\hline & 50 & $3.7 \pm 0.8^{b}$ & $6.2 \pm 1.1^{\mathrm{b}}$ & $6.4 \pm 2.1^{\mathrm{b}}$ & $5.8 \pm 1.5^{\mathrm{b}}$ & $5 \pm 1.7^{b}$ \\
\hline \multirow[t]{3}{*}{ Active mitochondria (\%)* } & 0 & $24.5 \pm 3.9$ & $20.9 \pm 2.1$ & $23.5 \pm 3.6$ & $15.8 \pm 1.7$ & $14.7 \pm 2$ \\
\hline & 10 & $25.5 \pm 5.1$ & $27.8 \pm 4.7$ & $28.5 \pm 5.3$ & $23.1 \pm 3.9$ & $15.1 \pm 3$ \\
\hline & 50 & $25.4 \pm 5.7$ & $27.5 \pm 4.1$ & $25.7 \pm 3.6$ & $15.1 \pm 1.6$ & $12 \pm 1.6$ \\
\hline \multirow[t]{3}{*}{ Intracellular Ca (MFI) } & 0 & $10.5 \pm 1.1^{\mathrm{a}}$ & $9.1 \pm 0.5$ & $8.7 \pm 0.5^{\mathrm{a}}$ & $8.6 \pm 0.6$ & $8.6 \pm 0.7$ \\
\hline & 10 & $8.7 \pm 0.6^{\mathrm{ab} \alpha \beta}$ & $9.2 \pm 0.6^{\alpha}$ & $7.9 \pm 0.6^{\mathrm{b} \beta}$ & $8 \pm 0.6^{\beta}$ & $8.9 \pm 0.8^{\alpha \beta \beta}$ \\
\hline & 50 & $7.9 \pm 0.8^{b}$ & $9 \pm 0.8$ & $8.3 \pm 0.6^{\mathrm{ab}}$ & $8.8 \pm 0.6$ & $9.4 \pm 0.7$ \\
\hline
\end{tabular}

Values are presented as mean \pm SEM, with significant differences among plasma concentrations (rows) indicated by Latin letters and significant differences among times (columns) indicated by Greek letters.

Abbreviation: MFI, median fluorescence intensity.

* The proportion of spermatozoa with active mitochondria was only affected by incubation time (no interaction), with results at 4 hours being significantly lower than those at 0,1 , and 2 hours.

( $\mathrm{P}<0.05$ ), following the loss of sperm viability, but the samples incubated with $50 \%$ seminal plasma tended to a faster increase (Table 2). Interestingly, this increase was not only due to a higher mortality in this treatment, but also due to a higher proportion of viable spermatozoa with reacted acrosomes (significantly $>0 \%$ and $10 \%$ at all times, $\mathrm{P}<0.05$ ).

Intracellular ROS concentration (Table 3) increased during the first hour of incubation in samples treated with $10 \%$ and $50 \%$ seminal plasma. This increase continued at 2 hours in samples treated with 50\% seminal plasma, which achieved significantly higher values than the other two treatments up to the end of the experiment $(\mathrm{P}<0.01$, except for $50 \%$ vs. $10 \%$ at 3 hours, with $\mathrm{P}=0.015$ ). The increase in intracellular ROS was not accompanied by a decrease of 5-IAF fluorescence (free thiols on the cell surface). Contrarily, this signal increased with time in 50\% seminal plasma, being significantly $>0 \%$ and $10 \%$ samples at 2 hours onwards $(P<0.05)$.

The proportion of spermatozoa with active mitochondria was not significantly different among the treatments. This proportion decreased with time after 2 hours, with results at 4 hours being lower than at the beginning of the experiment $(P<0.001$; Table 2). Similarly, differences between treatments regarding intracellular $\mathrm{Ca}^{2+}$ concentration were very small. We only detected some fluctuations at 0 hour (samples with 50\% seminal plasma being lower than samples with no plasma) and at 2 hours (samples with 10\% seminal plasma being lower than samples with no plasma).

In order to better characterize the dynamics of the changes during the incubation, taking into account the between-samples variability, we carried out a PCA, complemented with a cluster analysis. The variables used in this analysis were as follows: proportion of spermatozoa with damaged acrosomes $(A)$; undamaged plasma membrane with normal permeability (V); increased membrane fluidity $(\mathrm{F})$; median fluorescence intensity corresponding to intracellular ROS (R); and presence of free thiols in the cell surface of viable spermatozoa (T). In all cases, based on Kaiser's criterion, two principal components were selected, which explained $>70 \%$ of the variance. The results of the PCA are displayed in Figures 2-6. In these plots, overplotted vectors represent the contributions of each individual variable to each principal component (PC1 and PC2). Ellipses include observations clustered together.

At 0 hour (Fig. 2), it is evident that observations were not well separated by the treatments $(0 \%, 10 \%$, or $50 \%$ seminal plasma), showing the heterogeneity of the thawed samples (samples grouped by male or by similar males). In fact, male 6 is shown as a "outlier" (high viability $-\mathrm{V}-$ with low 5-IAF-T-fluorescence and low acrosomal damage -A-). At $1 \mathrm{~h}$ (Fig. 3), while some observations were still

Table 3

Intracellular ROS and the levels of free thiols on the sperm surface during the incubation with different proportions of seminal plasma.

\begin{tabular}{|c|c|c|c|c|c|c|}
\hline \multirow[t]{2}{*}{ Variable } & \multirow[t]{2}{*}{ Plasma } & \multicolumn{5}{|c|}{ Hours of incubation } \\
\hline & & 0 & 1 & 2 & 3 & 4 \\
\hline \multirow[t]{3}{*}{ Intracellular ROS (MFI) } & 0 & $15.7 \pm 2.7$ & $16.6 \pm 1.5$ & $18.8 \pm 1.8^{\mathrm{a}}$ & $20.9 \pm 3.4^{\mathrm{a}}$ & $17.1 \pm 3.1^{\mathrm{a}}$ \\
\hline & 10 & $11.1 \pm 1.3^{\alpha}$ & $17.6 \pm 2.2^{\beta}$ & $19.7 \pm 1.5^{\mathrm{a} \beta}$ & $21.9 \pm 2.7^{\mathrm{a} \beta}$ & $21.4 \pm 4^{\mathrm{b} \beta}$ \\
\hline & 50 & $12 \pm 2.4^{\alpha}$ & $19 \pm 2.5^{\beta}$ & $31.5 \pm 6^{\mathrm{b} \gamma}$ & $26.6 \pm 4^{\mathrm{b} \beta \gamma}$ & $39.1 \pm 13.6^{\mathrm{b} \gamma}$ \\
\hline \multirow[t]{3}{*}{ Free thiols (cell surface) (MFI) } & 0 & $37.7 \pm 3.8$ & $37.6 \pm 6.1$ & $37 \pm 4.9^{\mathrm{a}}$ & $38.2 \pm 4.2^{\mathrm{a}}$ & $36.1 \pm 4.3^{\mathrm{a}}$ \\
\hline & 10 & $35.7 \pm 4$ & $38.1 \pm 5.6$ & $36.3 \pm 5.8^{a}$ & $38.9 \pm 4.9^{\mathrm{a}}$ & $36.5 \pm 5.4^{\mathrm{a}}$ \\
\hline & 50 & $38.1 \pm 6.2^{\alpha}$ & $40.6 \pm 5.5^{\alpha \beta}$ & $44 \pm 6^{\mathrm{b} \beta}$ & $44.8 \pm 4.5^{\mathrm{b} \beta}$ & $42.8 \pm 4.8^{\mathrm{b} \beta}$ \\
\hline
\end{tabular}

Values are presented as mean \pm SEM, with significant differences among plasma concentrations (rows) indicated by Latin letters and significant differences among times (columns) indicated by Greek letters.

Abbreviation: MFI, median fluorescence intensity. 


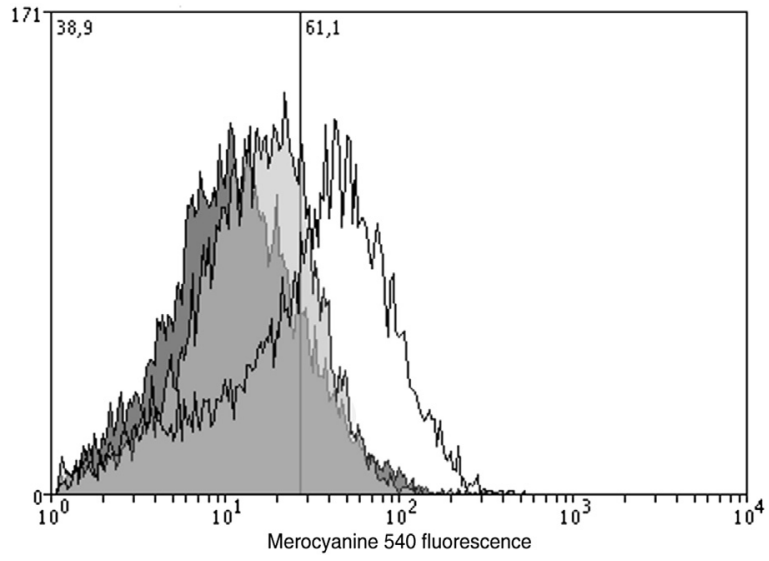

Fig. 1. Overlapped histograms corresponding to the merocyanine 540 fluorescence of live spermatozoa in three thawed samples: incubated with $0 \%$ (dark gray), 10\% (medium gray), or 50\% (light gray) seminal plasma for 2 hours. The fluorescence distribution in the sample incubated with $50 \%$ seminal plasma is clearly increased in a high proportion of spermatozoa.

being clustered together by male (grouping males $(1,2)$, $(3,6)$ and $(4,5,7))$, the effect of $50 \%$ seminal plasma started to be evident, with five out of seven observations belonging to this treatment being clustered together. That cluster was characterized by higher intracellular ROS (R), free thiols (T), and increased membrane fluidity (F) (although there was some variability within the cluster). At 2 hours (Fig. 4), this structure changed slightly, following a change in the multidimensional space. At 3 hours (Fig. 5), there was an increase in heterogeneity among the observations, reflected in the increased number of clusters, but observations associated to 50\% seminal plasma were grouped together in two clusters. These two clusters were characterized by increased membrane fluidity $(F)$, free thiols $(T)$, differing in viability (V) and intracellular ROS (R) (samples from males 3,6 , and 7 presenting higher values). Both clusters showed higher acrosomal damage (A) than clusters grouping samples from the $0 \%$ and $10 \%$ seminal plasma groups.

Finally, at 4 hours (Fig. 6), all the 50\% seminal plasma observations were clearly separated from the rest of the observations in a single cluster. These observations were characterized by higher membrane fluidity $(F)$, intracellular ROS (R), and acrosomal damage (A), although there was some heterogeneity regarding acrosomal damage (notice the direction of the A vector relative to the cluster shape).

This study has shown that the supplementation of thawed boar semen with 50\% seminal plasma caused a dramatic effect in the plasma membrane status, accompanied by other physiological changes, whereas these changes were not evident or much lower when adding $10 \%$ seminal plasma. These observations could help explaining the enhancing effect of seminal plasma on thawed semen fertility observed in other studies $[15,16,19]$. The protection that seminal plasma confers to boar spermatozoa against cold shock has been known for a long time [25]. The PSP-I/ PSP-II heterodimer (non-heparin-binding spermadhesins [26]) represent more than half of the seminal plasma proteins in the boar. This heterodimer seems to be responsible, at least in part, of the beneficial effects of seminal plasma on stressed spermatozoa (frozen-thawed [15,19], highly extended [27], and sorted [28]). Other components could be involved in important events, such as the modulation of the immune response in the uterus [29] or the formation of the oviductal reservoir [30]. Different studies have been defining these components and their effects in vivo and in vitro, which may be very different depending on the environment [31].

\section{Discussion}

analysis is shown in the dendrogram on the left, with each of the four clusters detected (mostly discriminated by male origin) enclosed by gray rectangles. The plot on the right shows the spatial distribution of the samples according to the first principal components, with ellipses grouping samples belonging to the same cluster. Samples are identified by male number and plasma proportion. Vectors show the relationship between each of the principal components and the original variables. A, acrosomal damage; F, membrane fluidity; M, mitochondrial activity; R, intracellular ROS; V, viability; T, free thiols. 

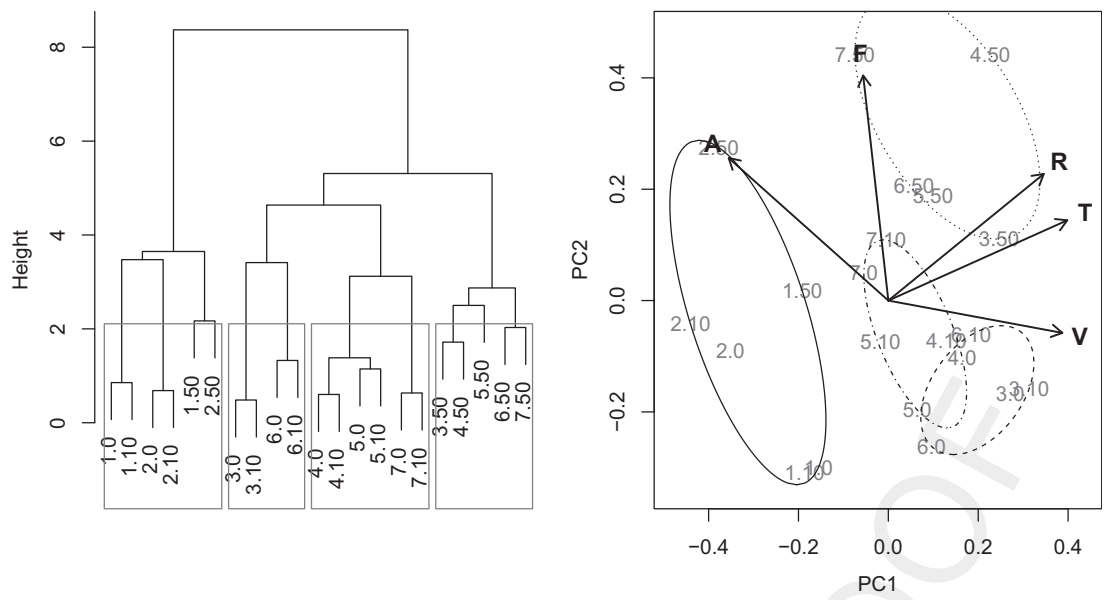

Fig. 3. Graphical description of the multidimensional study with the physiological variables obtained after 1 hour of incubation at $37^{\circ} \mathrm{C}$ (plot components are described in Fig. 2). The clustering has changed from the initial sampling (samples grouped by male), and a cluster containing most samples incubated with 50\% plasma emerges. This cluster is characterized by an increased proportion of reacted acrosomes (A), membrane fluidity (F), and, in some samples, by higher mitochondrial activity $(\mathrm{M})$.

Our study follows García et al. [19], who found a fertility improvement when supplementing thawed boar semen with $50 \%$ seminal plasma. The most noticeable effect that we have observed in our study is an important increase in membrane fluidity in the samples treated with $50 \%$ seminal plasma, as assessed by merocyanine 540 . These changes have been associated with capacitation events by several authors [32], although this has been put into question [33]. A modulation of capacitation by seminal plasma could be related to the results observed by García et al. [19]. However, we could not detect large shifts in the intracellular calcium concentration in any treatment, which are a clear indicator of capacitation [6]. Interestingly, we could not detect a conspicuous "increased membrane permeability" population in our analyses. This population is stained by YO-PRO-1, and it has been related to low fertility results in some species [34], although this relationship is not clear for boar semen [35]. We found that membrane changes in our experimental treatments could occur very quickly; thus, we could only detect a very low proportion of cells in the "transitional" state of increased membrane permeability.

Except for García et al. [19], previous studies on thawed boar spermatozoa have tested supplementation with seminal plasma up to $20 \%$ (most of them $10 \%$ ). For instance, Vadnais et al. [7] reported that $10 \%$ and $20 \%$ of seminal plasma reduced the proportion of capacitated spermatozoa (using the chlortetracycline assay) when frozen-thawed boar spermatozoa were incubated in a capacitation-supporting medium. However, when Vadnais et al. [7] used merocyanine 540 to assess the fluidity of the plasma membrane [9], they obtained no differences among samples frozen with and without $10 \%$ seminal plasma, resembling our results with that treatment. In the same study, no differences were reported among
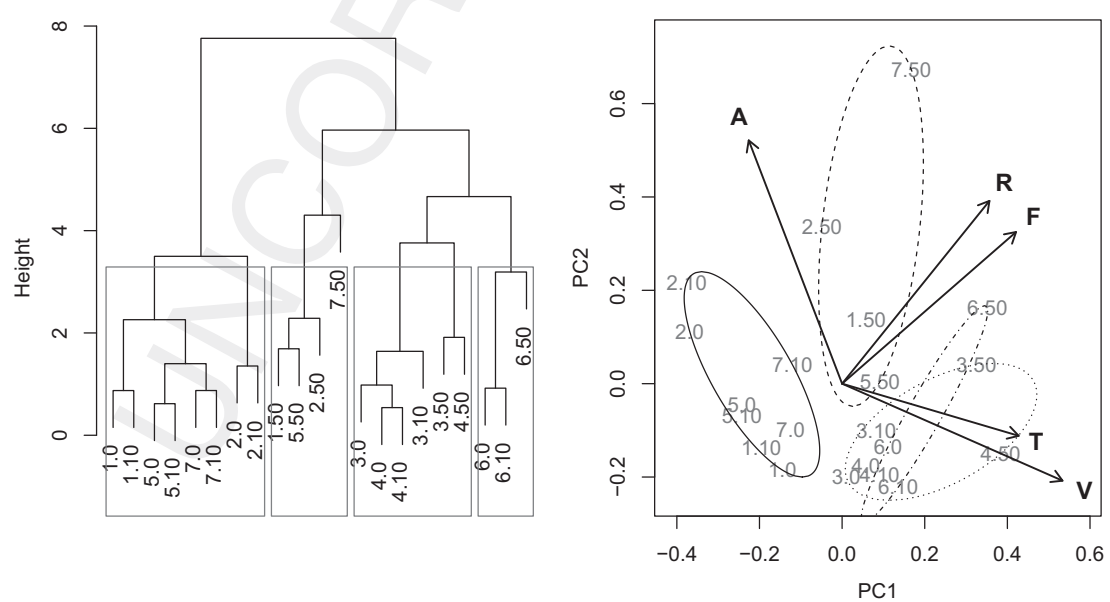

Fig. 4. Graphical description of the multidimensional study with the physiological variables obtained after 2 hours of incubation at $37{ }^{\circ} \mathrm{C}$ (plot components are described in Fig. 2). Samples incubated with no plasma or 10\% seminal plasma has formed a compact cluster. Samples incubated with $50 \%$ seminal plasma have been included in a more heterogeneous cluster characterized mainly by higher membrane fluidity (F) and higher intracellular ROS (R). 

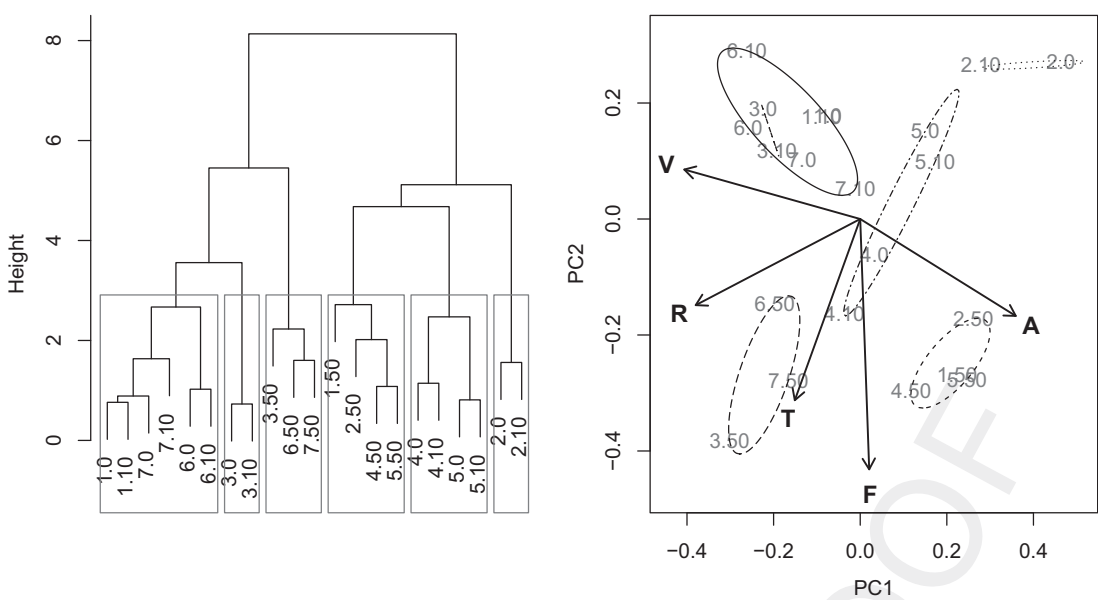

Fig. 5. Graphical description of the multidimensional study with the physiological variables obtained after 3 hours of incubation at $37{ }^{\circ} \mathrm{C}$ (plot components are described in Fig. 2). Samples incubated with 50\% seminal plasma remain grouped in the same cluster, showing increased reacted acrosomes (A), membrane fluidity (F), free thiols (T), and lower mitochondrial activity (M).

sperm samples assayed for Annexin V just after thawing, after incubating in noncapacitating medium or after incubating with $10 \%$ seminal plasma. The Annexin V assay allows to recognize spermatozoa with "apoptotic-like" features, and, therefore, these results seem to agree with our YO-PRO-1 results regarding the viable "apoptotic" subpopulation.

Interestingly, the membrane changes in our samples incubated with $50 \%$ seminal plasma were accompanied by an increase of intracellular ROS and by an increase in the proportion of spermatozoa with damaged or reacted acrosomes. These observations deserve further research to determine if there is a causal relationship among them. Our data suggest that a small population of spermatozoa (no necessarily the same) respond very quickly to the exposure to $50 \%$ seminal plasma, undergoing the acrosome reaction (viable acrosome-reacted population increasing significantly at 0 hour) and an increase in membrane fluidity (nonsignificant increase at 0 hour). In fact, this might be a very dynamic process, with viable spermatozoa undergoing these changes and thus being detected as either merocyanine-positive or acrosome-reacted (but still viable), while other spermatozoa from these populations are dying. However, this dynamics seemed to be different for both processes. The dying rate in the acrosome-reacted population seemed to be similar to the acrosome-reaction rate (as deduced by the constant and small proportion of the viable acrosome-reacted population). However, in the case of the merocyanine-positive population, the changes causing an increase of membrane fluidity in 50\% seminal plasma seemed to spread throughout the population of viable spermatozoa, resulting in the considerable increase of merocyanine-positive/viable spermatozoa ratio with advancing incubation time.

Of course, if $50 \%$ seminal plasma were to cause an important oxidative stress to thawed boar spermatozoa, we could expect an important decrease in fertility due to the

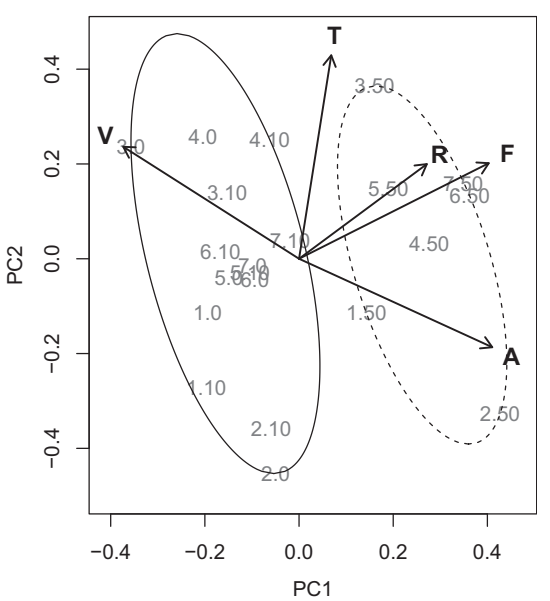

Fig. 6. Graphical description of the multidimensional study with the physiological variables obtained after 4 hours of incubation at $37^{\circ} \mathrm{C}$ (plot components are described in Fig. 2). Samples incubated with $50 \%$ seminal plasma are clearly separated from the rest of the samples, mainly by the proportion of reacted acrosomes (A), membrane fluidity (F), and, secondarily, intracellular ROS (R). 
954 sensitivity of boar spermatozoa [36], but this is not supported by the results of García et al. [19]. In fact, the amount of free thiols in the sperm surface was not reduced by the ROS increase. This paradoxical finding could be explained by two facts: first, seminal plasma also contains many antioxidants, which could contribute to its beneficial effects [37]; and second, the ROS increase might be within the physiological levels, not causing oxidative stress. It is known that free radicals have a role as second messengers during physiological events in spermatozoa [38], being important activators of capacitation and other events. This stimulatory effect, although detrimental for many spermatozoa, could be positive after the insemination, contributing to the formation of the oviductal reservoir and improving the fertilizing potential of at least a subpopulation of spermatozoa. Whereas several authors have highlighted the importance of seminal plasma for the formation of the sperm oviductal reservoir and gamete interaction [30,39,40], others have noted a lack of effects of supplementing boar spermatozoa with only $10 \%$ seminal plasma $[17,18]$. Subsequent studies might investigate if there is a relationship between the formation of the oviductal reservoir and the capacity of seminal plasma to stimulate ROS levels, which might explain the differences with studies using a low concentration of seminal plasma [15]. Nevertheless, we cannot discard other positive effects of using high concentrations, such as an improvement of membrane condition (increase of free thiols) or a direct effect on the female genital tract [26,41].

Even though we detected the increase in reacted acrosomes and membrane fluidity earlier than the rise in intracellular free radicals, we cannot discard a role for free radicals from the beginning of the experiment. The activation of a small sperm subpopulation at 0 hour could have passed unnoticed, and the significant increase at 2 hours could reflect the generalization of an effect starting in a sensitive subpopulation immediately after adding the seminal plasma. We have to highlight that such a change in intracellular ROS, after adding seminal plasma to thawed boar semen, has not been reported before. Indeed, it could help to explain some of the effects of seminal plasma in boar spermatozoa, both positive and negative, and the differences that we have found between males in the clustering analysis. These between-male differences could be explained in terms of the importance of a sperm subpopulation susceptible to producing and/or responding to ROS, but this hypothesis must be tested in other studies. Several studies [42-44] have highlighted the differences between males regarding not only sperm quality or freezability but also the response to different stimuli. Moreover, there are individual differences regarding the composition of seminal plasma [45], implying that the effects of a batch of seminal plasma could vary depending on its source. These differences might depends also on the breed. In this study, we aimed at exploring the individual variation of each sample by means of a multidimensional analysis, but analyzing the effect of males of different quality or different breeds was beyond our objectives (we used too few males per breed, and the males used both for obtaining semen and seminal plasma were selected by high semen quality and high fertility). Nevertheless, the multidimensional study showed that the $50 \%$ seminal plasma treatment not only affected the sperm samples from all males but also that there were individual differences in that response. This study might be enhanced by following two lines focused in the between-male and between-breed differences, aimed at the practical application of these findings: analyzing the response of spermatozoa from heterogeneous sources treated with a single batch of seminal plasma and analyzing the response of spermatozoa from homogeneous sources treated with heterogeneous batches of seminal plasma.

The effect of seminal plasma on spermatozoa is very complex, and its application often results in contradictory results depending on the type of samples (fresh, cooled, thawed, and sorted) [31]. These differences could explain the variability found among studies. Many components of the seminal plasma (especially spermadhesins) interact with the plasma membrane [41]. However, the effect of a high proportion of seminal plasma or a high concentration of spermadhesins added after thawing have been scarcely studied. Freezing boar sperm in the presence of $>25 \%$ seminal plasma worsened post-thawing results [46], whereas $25 \%$ or lower concentrations improved semen quality and resilience after thawing. In that study, the authors also investigated the effect of adding seminal plasma after thawing, concluding that the main effects of seminal plasma were exerted during the freezing process, having a minor role if added after thawing. However, they did not test concentrations above $12.5 \%$. Our results with $10 \%$ seminal plasma seem to agree with that study, although we could detect a moderate ROS increase with $10 \%$ seminal plasma, which might be involved in the positive effects noted by other authors [7]. Thus, spermatozoa were, in fact, affected by the incubation with $10 \%$ seminal plasma, even if no differences were found using other probes. It might be interesting to test concentrations between $20 \%$ and $50 \%$, in order to detect the critical concentration causing the effects noticed with $50 \%$ in our study. Moreover, although it might be of limited utility in the practice, studying concentrations beyond 50\% could help to understand the effects of lower seminal plasma concentrations in thawed semen.

Moreover, García et al. [19] found a beneficial effect in sperm viability when using $50 \%$ seminal plasma, even after 4 hours of incubation. In our study, viability at 4 hours was lower for $50 \%$ seminal plasma (however, overall viability was higher in our experiment). Apart from using a different methodology (fluorochromes and microscopy vs. cytometry), we attribute the lower viability at 4 hours to the higher membrane fluidity induced by incubating with $50 \%$ seminal plasma, which would render the spermatozoa more vulnerable to the dilution before the flow cytometry analysis (which was absent in the protocols by García et al. [19]). This effect was detected in other experiments [47], but not in other occasions when high dilutions were not applied [48]. Nevertheless, we do not think that our viability results contradict the fertility results of $50 \%$ seminal plasma. First, samples apparently more vulnerable, which yield lower viability in some analyses, might yield better fertility results [49]. The fertility potential depends on many factors, and even if a sample seems to contain a subpopulation of vulnerable spermatozoa, it might also contain another subpopulation of high-quality 
spermatozoa, which might be responsible of the fertility of the whole sample, as some authors have proposed [50,51]. Second, we have observed these differences in viability at 4 hours of incubation, whereas other changes (such as membrane fluidity) seemed to be induced much earlier. This is important because inseminated spermatozoa are in a very different environment than incubated spermatozoa. Indeed, other early effect that we have observed is a higher viability at 2 hours in 50\% seminal plasma, which might be more relevant for the practical use in vivo. The purpose of incubating the samples was not replicating the in vivo environment in the oviduct, but rather enhances small changes produced by the treatments to help to interpret their effects.

In conclusion, although $10 \%$ seminal plasma produced limited effects in the physiology of thawed boar spermatozoa, $50 \%$ seminal plasma caused noticeable changes. We propose that a high proportion of seminal plasma could stimulate the spermatozoa, possibly through the production of free radicals starting from a small sensitive subpopulation. These events would benefit the overall performance of the sperm sample, despite undesirable effects in more sensitive cells (acrosomal reaction and subsequent loss of viability). These hypotheses could be tested in future studies, using more specific approaches.

\section{Uncited Figure}

\section{Q6 Figure 1.}

\section{Acknowledgments}

This work has been supported by project DPI200908424 (Diputación de León, Spain). Rocío Fernández-Gago was supported by a PhD grant (Junta de Castilla y León, Spain), and F. Martínez-Pastor was supported by the Ramón y Cajal program (RYC-2008-02560, Ministry of Science and Innovation, Spain). The authors thank CENTROTEC (León, Spain) for housing the animals and providing the semen samples.

\section{References}

[1] Bailey JL, Lessard C, Jacques J, Brèque C, Dobrinski I, Zeng W, et al. Cryopreservation of boar semen and its future importance to the industry. Theriogenology 2008;70:1251-9.

[2] Grossfeld R, Sieg B, Struckmann C, Frenzel A, Maxwell WMC, Rath D. New aspects of boar semen freezing strategies. Theriogenology 2008;70:1225-33.

[3] Roca J, Rodríguez-Martínez H, Vázquez JM, Bolarín A, Hernández M, Saravia F, et al. Strategies to improve the fertility of frozen-thawed boar semen for artificial insemination. Soc Reprod Fertil Suppl 2006; 62:261-75.

[4] Bolarín A, Roca J, Rodríguez-Martínez H, Hernández M, Vázquez JM, Martínez EA. Dissimilarities in sows' ovarian status at the insemination time could explain differences in fertility between farms when frozen-thawed semen is used. Theriogenology 2006;65:66980.

[5] Bolarín A, Hernández M, Vázquez JM, Rodríguez-Martínez H, Martínez EA, Roca J. Use of frozen-thawed semen aggravates the summer-autumn infertility of artificially inseminated weaned sows in the Mediterranean region. J Anim Sci 2009;87:3967-75.

[6] Green CE, Watson PF. Comparison of the capacitation-like state of cooled boar spermatozoa with true capacitation. Reproduction 2001;122:889-98.
[7] Vadnais ML, Kirkwood RN, Specher DJ, Chou K. Effects of extender, incubation temperature, and added seminal plasma on capacitation of cryopreserved, thawed boar sperm as determined by chlortetracycline staining. Anim Reprod Sci 2005;90:347-54.

[8] Vadnais ML, Roberts KP. Effects of seminal plasma on coolinginduced capacitative changes in boar sperm. J Androl 2007;28: $416-22$.

[9] Vadnais ML, Althouse GC. Characterization of capacitation, cryoinjury, and the role of seminal plasma in porcine sperm. Theriogenology 2011;76:1508-16.

[10] Garner DL, Thomas CA, Gravance CG, Marshall CE, DeJarnette JM, Allen $\mathrm{CH}$. Seminal plasma addition attenuates the dilution effect in bovine sperm. Theriogenology 2001;56:31-40.

[11] Martínez-Pastor F, Anel L, Guerra C, Álvarez M, Soler AJ, Garde JJ, et al. Seminal plasma improves cryopreservation of Iberian red deer epididymal sperm. Theriogenology 2006;66:1847-56.

[12] de Andrade AFC, Zaffalon FG, Celeghini ECC, Nascimento J, Bressan FF, Martins SMMK, et al. Post-thaw addition of seminal plasma reduces tyrosine phosphorylation on the surface of cryopreserved equine sperm, but does not reduce lipid peroxidation. Theriogenology 2012;77:1866-1872.e1-3.

[13] Rovegno M, Feitosa WB, Rocha AM, Mendes CM, Visintin JA, D'Avila Assumpção MEO. Assessment of post-thawed ram sperm viability after incubation with seminal plasma. Cell Tissue Bank 2013;14: 333-9.

[14] Suzuki K, Asano A, Eriksson B, Niwa K, Nagai T, Rodríguez-Martínez H. Capacitation status and in vitro fertility of boar spermatozoa: effects of seminal plasma, cumulus-oocyte complexes-conditioned medium and hyaluronan. Int J Androl 2002;25:84-93.

[15] Okazaki T, Abe S, Yoshida S, Shimada M. Seminal plasma damages sperm during cryopreservation, but its presence during thawing improves semen quality and conception rates in boars with poor post-thaw semen quality. Theriogenology 2009;71:491-8.

[16] Okazaki T, Shimada M. New strategies of boar sperm cryopreservation: development of novel freezing and thawing methods with a focus on the roles of seminal plasma. Anim Sci J 2012;83:623-9.

[17] Abad M, Sprecher DJ, Ross P, Friendship RM, Kirkwood RN. Effect of sperm cryopreservation and supplementing semen doses with seminal plasma on the establishment of a sperm reservoir in gilts. Reprod Domest Anim 2007;42:149-52.

[18] Abad M, García JC, Sprecher DJ, Cassar G, Friendship RM, Buhr MM, et al. Effect of insemination-ovulation interval and addition of seminal plasma on sow fertility to insemination of cryopreserved sperm. Reprod Domest Anim 2007;42:418-22.

[19] García JC, Domínguez JC, Peña FJ, Alegre B, Gonzalez R, Castro MJ, Habing GG, Kirkwood RN. Thawing boar semen in the presence of seminal plasma: effects on sperm quality and fertility. Anim Reprod Sci 2010;119:160-5.

[20] Eriksson BM, Rodríguez-Martínez $\mathrm{H}$. Effect of freezing and thawing rates on the post-thaw viability of boar spermatozoa frozen in flatpacks and maxi-straws. Anim Reprod Sci 2000;63:205-20.

[21] Peláez J, Breininger E, Alegre B, Peña FJ, Domínguez JC. In vitro evaluation of the quality and fertilizing capacity of boar semen frozen in $0.25 \mathrm{ml}$ straws. Reprod Domest Anim 2006;41:153-61.

[22] Domínguez-Rebolledo AE, Martínez-Pastor F, Bisbal AF, RosSantaella JL, García-Álvarez O, Maroto-Morales A, et al. Response of thawed epididymal red deer spermatozoa to increasing concentrations of hydrogen peroxide, and importance of individual male variability. Reprod Domest Anim 2011;46:393-403.

[23] R Development Core Team. R: a language and environment for statistical computing. Vienna, Austria: R Foundation for Statistical Computing; 2012.

[24] Kaufman L, Rousseeuw P. Finding groups in data: an introduction to cluster analysis. New York: Wiley; 1990.

[25] Pursel VG, Johnson LA, Schulman LL. Effect of dilution, seminal plasma and incubation period on cold shock susceptibility of boar spermatozoa. J Anim Sci 1973;37:528-31.

[26] Caballero I, Vázquez JM, García EM, Parrilla I, Roca J, Calvete JJ, et al. Major proteins of boar seminal plasma as a tool for biotechnological preservation of spermatozoa. Theriogenology 2008;70:1352-5.

[27] Caballero I, Vázquez JM, García EM, Roca J, Martínez EA, Calvete JJ, et al. Immunolocalization and possible functional role of PSP-I/PSPII heterodimer in highly extended boar spermatozoa. J Androl 2006; 27:766-73.

[28] García EM, Vázquez JM, Parrilla I, Calvete JJ, Sanz L, Caballero I, et al. Improving the fertilizing ability of sex sorted boar spermatozoa. Theriogenology 2007;68:771-8.

[29] Assreuy AMS, Alencar NMN, Cavada BS, Rocha-Filho DR, Feitosa RFG, Cunha FQ et al. Porcine spermadhesin PSP-I/PSP-II stimulates 
macrophages to release a neutrophil chemotactic substance: modulation by mast cells. Biol Reprod 2003;68:1836-41.

[30] Ekhlasi-Hundrieser M, Gohr K, Wagner A, Tsolova M, Petrunkina A, Töpfer-Petersen E. Spermadhesin aqn1 is a candidate receptor molecule involved in the formation of the oviductal sperm reservoir in the pig. Biol Reprod 2005;73:536-45.

[31] Caballero I, Parrilla I, Almiñana C, del Olmo D, Roca J, Martínez EA, et al. Seminal plasma proteins as modulators of the sperm function and their application in sperm biotechnologies. Reprod Domest Anim 2012;47(Suppl 3):12-21.

[32] Martínez-Pastor F, Mata-Campuzano M, Álvarez-Rodríguez M, Álvarez M, Anel L, de Paz P. Probes and techniques for sperm evaluation by flow cytometry. Reprod Domest Anim 2010;45(Suppl 2):67-78.

[33] Muratori M, Porazzi I, Luconi M, Marchiani S, Forti G, Baldi E. Annexin $\mathrm{V}$ binding and merocyanine staining fail to detect human sperm capacitation. J Androl 2004;25:797-810.

[34] Anzar M, He L, Buhr MM, Kroetsch TG, Pauls KP. Sperm apoptosis in fresh and cryopreserved bull semen detected by flow cytometry and its relationship with fertility. Biol Reprod 2002;66: 354-60.

[35] Trzcińska M, BryŃa M, Smorag Z. Apoptotic-like changes in the spermatozoa of fresh and stored boar semen and the quality of embryos produced in vivo. Anim Reprod Sci 2011;124:90-7.

[36] Awda BJ, Mackenzie-Bell M, Buhr MM. Reactive oxygen species and boar sperm function. Biol Reprod 2009;81:553-61.

[37] Leahy T, Celi P, Bathgate R, Evans G, Maxwell WMC, Marti JI. Flowsorted ram spermatozoa are highly susceptible to hydrogen peroxide damage but are protected by seminal plasma and catalase. Reprod Fertil Dev 2010;22:1131-40.

[38] O'Flaherty C, de Lamirande E, Gagnon C. Positive role of reactive oxygen species in mammalian sperm capacitation: triggering and modulation of phosphorylation events. Free Radic Biol Med 2006; 41:528-40.

[39] Muiño-Blanco T, Pérez-Pé R, Cebrián-Pérez JA. Seminal plasma proteins and sperm resistance to stress. Reprod Domest Anim 2008; 43(Suppl 4):18-31.

[40] Caballero I, Vázquez JM, Mayor GM, Almiñana C, Calvete JJ, Sanz L, et al. PSP-I/PSP-II spermadhesin exert a decapacitation effect on highly extended boar spermatozoa. Int J Androl 2009; 32:505-13.
[41] Rodríguez-Martínez H, Kvist U, Ernerudh J, Sanz L, Calvete JJ. Seminal plasma proteins: what role do they play? Am J Reprod Immunol 2011;66(Suppl 1):11-22.

[42] Thurston LM, Siggins K, Mileham AJ, Watson PF, Holt WV. Identification of amplified restriction fragment length polymorphism markers linked to genes controlling boar sperm viability following cryopreservation. Biol Reprod 2002;66:545-54.

[43] Henning H, Petrunkina AM, Harrison RAP, Waberski D. Bivalent response to long-term storage in liquid-preserved boar semen: a flow cytometric analysis. Cytometry A 2012;81:576-87.

[44] Parrilla I, del Olmo D, Sijses L, Martinez-Alborcia MJ, Cuello C, Vazquez JM, et al. Differences in the ability of spermatozoa from individual boar ejaculates to withstand different semen-processing techniques. Anim Reprod Sci 2012;132:66-73.

[45] Dyck MK, Foxcroft GR, Novak S, Ruiz-Sanchez A, Patterson J, Dixon WT. Biological markers of boar fertility. Reprod Domest Anim 2011;46(Suppl 2):55-8.

[46] Gómez-Fernández J, Gómez-Izquierdo E, Tomás C, GonzálezBulnes A, Sánchez-Sánchez R, de Mercado E. Inclusion of seminal plasma in sperm cryopreservation of Iberian pig. Anim Reprod Sci 2012;130:82-90.

[47] Tamayo-Canul J, Alvarez M, López-Urueña E, Nicolas M, MartinezPastor F, Anel E, et al. Undiluted or extended storage of ram epididymal spermatozoa as alternatives to refrigerating the whole epididymes. Anim Reprod Sci 2011;126:76-82.

[48] Martínez AF, Martínez-Pastor F, Alvarez M, Fernández-Santos MR, Esteso MC, de Paz P, et al. Sperm parameters on Iberian red deer: electroejaculation and post-mortem collection. Theriogenology 2008;70:216-26.

[49] García-Alvarez O, Maroto-Morales A, Martínez-Pastor F, Garde JJ, Ramón M, Fernández-Santos MR, et al. Sperm characteristics and in vitro fertilization ability of thawed spermatozoa from black manchega ram: electroejaculation and postmortem collection. Theriogenology 2009;72:160-8.

[50] Holt WV, Harrison RAP. Bicarbonate stimulation of boar sperm motility via a protein kinase A-dependent pathway: between-cell and between-ejaculate differences are not due to deficiencies in protein kinase A activation. J Androl 2002;23:557-65.

[51] Martínez-Pastor F, Tizado EJ, Garde JJ, Anel L, de Paz P. Statistical series: opportunities and challenges of sperm motility subpopulation analysis. Theriogenology 2011;75:783-95. 